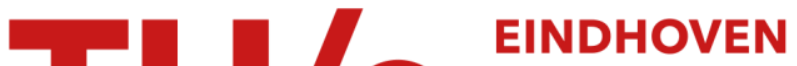 UNIVERSITY OF TECHNOLOGY
}

\section{Role of thermal hopping and homogeneous broadening on the spectral characteristics of quantum dot lasers}

\section{Citation for published version (APA):}

Markus, A., Rossetti, M., Calligari, V., Chen, J. X., \& Fiore, A. (2005). Role of thermal hopping and homogeneous broadening on the spectral characteristics of quantum dot lasers. Journal of Applied Physics, 98(10), 104506-1/8. [104506]. https://doi.org/10.1063/1.2133932

DOI:

$10.1063 / 1.2133932$

Document status and date:

Published: 01/01/2005

\section{Document Version:}

Publisher's PDF, also known as Version of Record (includes final page, issue and volume numbers)

\section{Please check the document version of this publication:}

- A submitted manuscript is the version of the article upon submission and before peer-review. There can be important differences between the submitted version and the official published version of record. People interested in the research are advised to contact the author for the final version of the publication, or visit the $\mathrm{DOI}$ to the publisher's website.

- The final author version and the galley proof are versions of the publication after peer review.

- The final published version features the final layout of the paper including the volume, issue and page numbers.

Link to publication

\section{General rights}

Copyright and moral rights for the publications made accessible in the public portal are retained by the authors and/or other copyright owners and it is a condition of accessing publications that users recognise and abide by the legal requirements associated with these rights.

- Users may download and print one copy of any publication from the public portal for the purpose of private study or research.

- You may not further distribute the material or use it for any profit-making activity or commercial gain

- You may freely distribute the URL identifying the publication in the public portal.

If the publication is distributed under the terms of Article 25fa of the Dutch Copyright Act, indicated by the "Taverne" license above, please follow below link for the End User Agreement:

www.tue.nl/taverne

Take down policy

If you believe that this document breaches copyright please contact us at:

openaccess@tue.nl

providing details and we will investigate your claim. 


\title{
Role of thermal hopping and homogeneous broadening on the spectral characteristics of quantum dot lasers
}

\author{
A. Markus, ${ }^{\text {a) }}$ M. Rossetti, V. Calligari, J. X. Chen, ${ }^{\text {b) }}$ and A. Fiore ${ }^{\text {c) }}$ \\ Ecole Polytechnique Fédérale de Lausanne (EPFL), Institute of Quantum Electronics and Photonics, \\ CH-1015 Lausanne, Switzerland
}

(Received 13 April 2005; accepted 10 October 2005; published online 29 November 2005)

\begin{abstract}
The effect of thermal hopping and homogeneous broadening on the lasing spectra and carrier distribution in quantum dot lasers is investigated by measuring the spontaneous emission spectra through a top-contact window. Two intriguing features are observed, i.e., a strong redshift of the excited-state lasing peak as compared to the spontaneous emission spectrum and the absence of population clamping at threshold. These features are interpreted on the basis of a rate-equation model taking into account energy-broadening mechanisms and thermal coupling among quantum dots. The good agreement between model and experiment allows us to estimate the homogeneous broadening and capture time, two key parameters for the understanding and proper design of quantum dot lasers. (C) 2005 American Institute of Physics. [DOI: 10.1063/1.2133932]
\end{abstract}

\section{INTRODUCTION}

Laser diodes based on semiconductor quantum dots (QDs) have attracted much attention due to various performance enhancements predicted ${ }^{1}$ for a zero-dimensional (0D) system. Subsequently, ultralow threshold current densities ${ }^{2}$ combined with decreased temperature sensitivity, ${ }^{3,4}$ highmodulation bandwith, ${ }^{4}$ and low chirp ${ }^{5}$ were reported for these devices. Moreover, the underlying physics differs remarkably from conventional quantum-well (QW) devices, which leads to a number of peculiar effects. For example, the coexistence of two lasing lines, which correspond to two different electronic transitions, has been reported ${ }^{6}$ and ascribed to the relatively slow intraband relaxation.

Yet the description of QDs as isolated 0D systems is not very accurate. More precisely, the active region of a QD laser is composed of an ensemble of OD structures, which are thermally coupled by a two-dimensional (2D) wetting-layer (WL) continuum. The QDs in the ensemble are slightly different in geometry and/or composition, which results in a dispersion of their electronic structure, as manifested in an inhomogeneously broadened spontaneous emission (SE) spectrum.

This paper focuses on the interaction among different QDs in a QD laser, either by (1) carrier transfer or by (2) homogeneous energy broadening. Intuitively carrier transfer among QDs can only happen through two mechanisms: tunneling between adjacent QDs, which is expected to be weak due to the large spatial distance and thus is neglected here, and thermal escape into the WL and subsequent capture into a different QD. Furthermore, QDs with transition energies within the homogeneous broadening can interact with the same photon bath, resulting in an indirect interaction. The

\footnotetext{
${ }^{a)}$ Electronic mail: alexander.markus@epfl.ch

${ }^{b)}$ Present address: Bell Laboratories, Lucent Technologies, Inc., 600 Mountain Ave., Murray Hill, NJ 07974.

${ }^{c)}$ Permanent address: Institute of Photonics and Nanotechnology, CNR, Via del Cineto Romano 42, 00156 Rome, Italy.
}

impact of these two mechanisms on the operation of QD lasers has been addressed mostly on the theoretical side, with emphasis on the transition of nonequilibrium to quasiequilibrium carrier distributions ${ }^{7-10}$ as well as the transition from broadband multimode lasing emission to a narrow lasing line. $^{7,11-13}$ This paper presents a detailed experimental investigation of carrier processes in QD lasers around and above the lasing threshold. Fitting these results with a numerical model allows a quantitative estimation of the crucial material parameters.

For this purpose both the spectral output from the laser facets and SE spectra are investigated. From the laser facets, the lasing spectrum and therefore information on the gain spectrum can be derived. On the other hand, the unamplified SE spectrum above the lasing threshold is of highest interest, since conclusions about the actual level populations can be drawn. Light detection from a window in the top-contact stripe of the device is a technique often exploited to gain experimental access to the SE spectrum. ${ }^{10,14}$ This way the population clamping at threshold can be investigated. In addition, the spectral distribution of carriers within the inhomogeneously broadened QD ensemble can be studied and linked to the spectral distribution of the lasing modes. This comparison allows us to draw conclusions about the degree of carrier localization within the inhomogeneously broadened QD ensemble, carrier thermalization, and the role of homogeneous broadening for the carrier-photon interaction. For example, a thermal carrier distribution down to $100 \mathrm{~K}$ has been evidenced in QD lasers emitting at $1.0 \mu \mathrm{m},{ }^{10}$ whereas no similar study yet exists for QD lasers emitting at longer wavelengths. The device investigated in this letter has an emission wavelength of $1.28 \mu \mathrm{m}$ on the ground-state (GS) transition, corresponding to much larger carrier localization energies, such that the establishment of thermal equilibrium within the QD system might be hindered.

The experimental data are then linked to a comprehensive rate-equation model, which takes into account both inhomogeneous and homogeneous energy broadening, and the 
impact of various parameters such as capture time, temperature, and homogeneous linewidth are discussed quantitatively.

The article is organized as follows: In Sec. II experimental results of spontaneous emission spectra versus stimulated emission spectra are discussed. In Sec. III a rate-equation model is developed and the impact of various parameters on the carrier distribution function is presented, as well as the consequences for the level population and gain spectrum. In Sec. IV the interaction with photons is implemented in the rate-equation model and the experimental spectra from Sec. II are reproduced. The impact of the homogeneous broadening is discussed. Finally, the main results are summarized in Sec. V.

\section{EXPERIMENTAL RESULTS}

The edge-emitting laser investigated in this section has been fabricated from the same epitaxial structure described in Ref. 6 in detail. It consists of a $\mathrm{Al}_{0.7} \mathrm{Ga}_{0.3} \mathrm{As} /$ $\mathrm{GaAs} / \mathrm{Al}_{0.7} \mathrm{Ga}_{0.3} \mathrm{As}$ waveguide with three QD layers, separated by $40 \mathrm{~nm}$. Lasers with as-cleaved facets and a cavity length $l>2.5 \mathrm{~mm}$ operate on the ground-state transition at $1.28 \mu \mathrm{m}$ and for $l<1.5 \mathrm{~mm}$ on the excited-state (ES) transition at $1.22 \mu \mathrm{m}$. For an intermediate cavity length (1.5 $<l<2.5 \mathrm{~mm}$ ) lasing takes place on both GS and ES transitions due to a combination of GS gain saturation and slow intraband relaxation time, as previously reported. ${ }^{6}$ In order to access the spontaneous emission spectrum during laser operation, $l=2$-mm-long devices were fabricated with $4 \times 10$ $\mu \mathrm{m}$-sized holes in the top-metal contact. The electroluminescence (EL) from these holes was then detected in a microphotoluminescence ( $\mu$-PL) setup, involving a Spex 1000M monochromator and an In-GaAs detector array. The EL from the facets was spectrally resolved by an optical spectrum analyzer.

Typical emission spectra from the laser facets at room temperature and in pulsed mode $(1 \mu$ s pulses, $1 \%$ duty cycle to minimize heating effects) are depicted in Fig. 1(a). At an injection current of $J=140 \mathrm{~A} / \mathrm{cm}^{2}$ the device starts lasing on the GS transition at $0.965 \mathrm{eV}$. With increasing bias the GS lasing line broadens and at $J=890 \mathrm{~A} / \mathrm{cm}^{2}$ a second lasing line appears at $1.025 \mathrm{eV}$, corresponding to the ES transition.

The corresponding spectra detected from a hole in the top-contact stripe are shown in Fig. 1(b). In this case the emission consists of both spontaneously emitted light and a fraction of the lasing mode intensity that is scattered by the hole. The latter is unintentional but quite useful to verify the lasing threshold and the position of the lasing peak. In fact, the observation of the same two-state lasing behavior from the facets and from the top-contact window confirms that this mechanism is intrinsic and not due to inhomogeneous carrier distribution along the cavity. The same behavior has been also observed on single transverse-mode lasers, ${ }^{6}$ providing further confirmation. The fraction of scattered light stemming from amplified spontaneous emission can be neglected, as confirmed by the absence of line narrowing in these spectra. Therefore, since apart from the lasing modes the spectra are composed of SE photons only, conclusions about the ac-

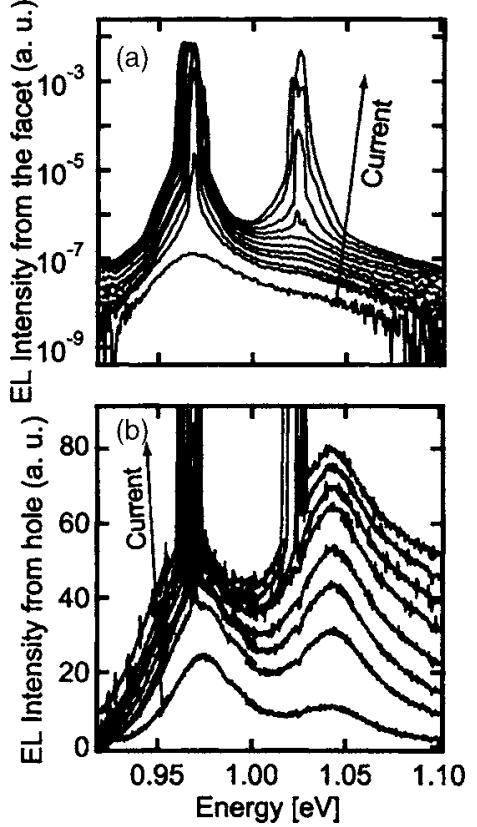

FIG. 1. Electroluminescence (EL) spectra from the facets in logarithmic scale (a) and from a window in the top-contact stripe in linear scale (b) at room temperature and at increasing injection current.

tual level population below and above the lasing threshold can be drawn. State filling becomes evident with increasing injection current. The GS SE is peaked at $0.97 \mathrm{eV}$, close to the spectral position of the lasing line. More strikingly, the ES SE peak is located at $1.045 \mathrm{eV}$ and is blueshifted by $20 \mathrm{meV}$ as compared to the position of the ES lasing line. Obviously gain peak and SE peak do not coincide, due to thermal carrier redistribution in the QD ensemble, as discussed in Sec. III in detail.

As a next step the quantitative dependence of the SE spectra on the injection current is studied. The GS and ES integrated SE intensities are proportional to the respective peak value of the SE spectrum, since the overlap of the two emissions is negligible. Figure 2(b) depicts the EL intensity close to the peak position of GS spontaneous emission $(E$ $=0.98 \mathrm{eV})$ and at the peak position of ES spontaneous emission $(E=1.045 \mathrm{eV})$ as a function of the injection current density. At GS threshold the increase of the GS spontaneous emission peak is strongly reduced and the slope further decreases at the ES threshold.

For both GS and ES a change in the slope of the SE intensity takes place at their respective threshold; however, no complete population clamping is observed, particularly for the ES. This behavior is in sharp contrast with the ideal picture of population clamping above the threshold. Even in QD lasers, a simple model neglecting carrier interactions in the QD ensemble ("single-mode" rate-equation model) results in a pinning of GS and ES populations at their respective threshold. ${ }^{6}$ In contrast to the experiment here GS and ES populations are continuously getting filled with increasing injection current and a complete population clamping is never observed.

Figure 2(a) shows light-current $(L-I)$ characteristics from the laser facet. GS and ES emissions have been detected 

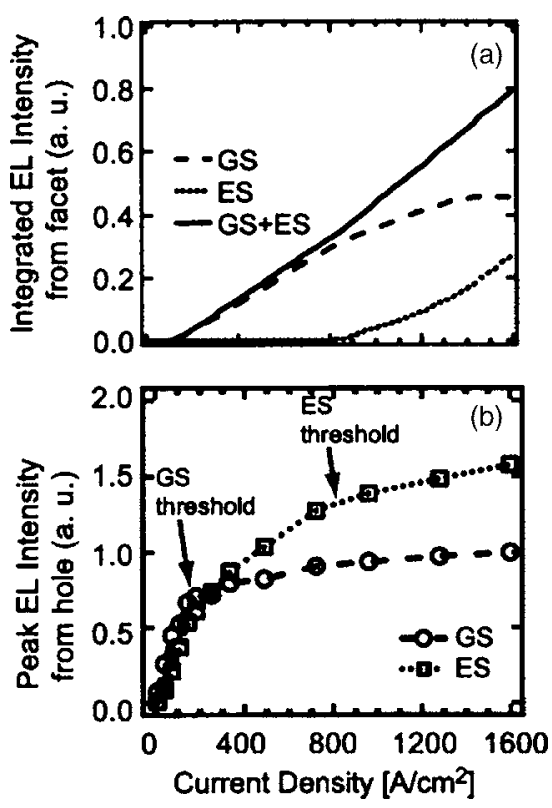

FIG. 2. (a) Light-current $(L-I)$ characteristics of GS and ES emissions detected from the laser facet. (b) Peak intensity of GS and ES spontaneous emission spectra in Fig. 1(b) as a function of the injection current density.

separately with high/low bandpass filters with a cuton/off wavelength at $1250 \mathrm{~nm}$. Obviously the GS differential efficiency gets increasingly suppressed above the ES threshold, but complete saturation of GS output power only occurs well above the ES threshold. In contrast, the single-mode model in Ref. 6 [Figs. 1(e) and 1(f)] predicts a complete saturation of GS emission at the ES threshold already. In Sec. IV this behavior will be related to the interplay between inhomogeneous and homogeneous energy broadening.

\section{CARRIER DISTRIBUTION FUNCTION}

In order to explain the spectral redshift of the ES lasing modes with respect to the ES population peak in Figs. 1(a) and 1(b), the carrier distribution function of the inhomogeneously broadened QD ensemble is calculated in this section. For the moment we describe the interaction of carriers in different QDs in the absence of photons. A similar study has been conducted in Ref. 9, where the discussion has been limited to GS population and gain function. Here we are interested in both GS and ES transitions and we adapt the model parameters to the experimentally found values. This allows us to quantitatively explain the experimental spectra.

The inhomogeneous energy broadening is treated by assuming a Gaussian distribution of the QD transition energies. Low-temperature PL spectra suggest a full width at half maximum (FWHM) of GS/ES energy distributions of $\mathrm{FWHM}_{\mathrm{GS}}=35 \mathrm{meV}$ and $\mathrm{FWHM}_{\mathrm{ES}}=45 \mathrm{meV}$. We discretize the energy axis in intervals $\Delta E_{\mathrm{GS}}, \Delta E_{\mathrm{ES}}$ around the GS/ES transition energies. All numerical calculations have been performed with an energy interval size of $\Delta E_{\mathrm{GS}} \approx 1 \mathrm{meV}$, $\Delta E_{\mathrm{ES}}=\Delta E_{\mathrm{GS}} \mathrm{FWHM}_{\mathrm{ES}} / \mathrm{FWHM}_{\mathrm{GS}}$. In the following we refer to $E_{i}$ as the GS transition energy of a given QD family. The corresponding ES transition energy of that QD family will be

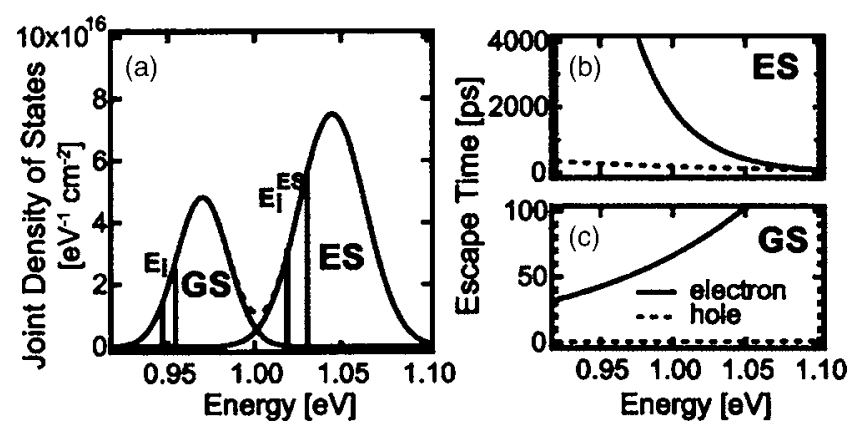

FIG. 3. (a) Density of states (DOS) of the modeled QD system. [(b) and (c)] Calculated escape times $\tau_{\mathrm{ES}}^{e, h\left(E_{i}\right)}$ from ES and $\tau_{\mathrm{GS}}^{e, h\left(E_{i}\right)}$ from GS, assuming a capture time $\tau_{c}=1 \mathrm{ps}$ and a relaxation time $\tau_{0}=10 \mathrm{ps}$.

labeled $E_{i}^{\mathrm{ES}}$ [see Fig. 3(a)]. The number of QDs with GS transition energies in a certain energy interval $E_{i} \pm \Delta E_{\mathrm{GS}} / 2$ can be calculated as

$$
N_{D}^{E_{i}}=\frac{N_{D}}{\sqrt{2 \pi} \sigma} \int_{E_{i}-\Delta E_{\mathrm{GS}} / 2}^{E_{i}+\Delta E_{\mathrm{GS}} / 2} \exp \left[\frac{-\left(E-E_{\mathrm{peak}}\right)^{2}}{2 \sigma^{2}}\right] d E,
$$

where $E_{\text {peak }}$ refers to the peak position and $\sigma$ to the variance of the GS transition energy distribution. By transmission electron microscopy (TEM) the total number of QDs has been estimated as $N_{D}=3 \times 10^{10} \mathrm{~cm}^{-2}$ per QD layer. The corresponding number of QDs $N_{D}^{\mathrm{ES}, E_{i}}$ with ES transition energies around $E_{i}$ can be derived the same way by accounting for a spectral shift of the ES peak energy $\left(E_{\text {peak }}+65 \mathrm{meV}\right)$, replacing $\sigma$ with the variance of the ES transition energies and the integration interval with $\Delta E_{\mathrm{ES}}$. QDs are assumed to confine electrons and holes in a twofold (spin-) degenerate GS and a fourfold degenerate ES due to an additional twofold degeneracy arising from the in-plane symmetry in the QD geometry. The resulting joint density of states (DOS) of the QD system [Fig. 3(a)] can be calculated as $\operatorname{DOS}\left(E_{i}\right)$ $=2\left(N_{D}^{E_{i}} / \Delta E_{\mathrm{GS}}\right)+4\left(N_{D}^{\mathrm{ES}, E_{i}} / \Delta E_{\mathrm{ES}}\right)$.

The carrier dynamics in the QD system is modeled in a rate-equation approach similar to the one presented in Refs. 6 and 15. However, rather than assuming eh-pairs that occupy corresponding energy states in their respective bands, in the model here electrons and holes are treated separately and the respective distribution functions are denoted as $f^{e}$ and $f^{h}$, respectively. It has been pointed out on Refs. 16 and 17 that such a treatment is crucial for a correct description of the temperature dependence of the threshold current. Thus electron and hole capture are uncorrelated and intraband electron-hole recombination is described by bimolecular recombination terms. As a result charge neutrality in different quantum dots is not imposed and thermal population of excited hole states is enhanced due to the more closely spaced valence-band states.

The respective GS/ES distribution functions $f_{\mathrm{GS}}^{e, h\left(E_{i}\right)}$ and $f_{\mathrm{ES}}^{e, h\left(E_{i}\right)}$ (corresponding to a QD family with a GS transition energy $E_{i}$ ) are described by a set of four rate equations each: 


$$
\begin{aligned}
\frac{d f_{\mathrm{GS}}^{e, h\left(E_{i}\right)}}{d t}= & \frac{2 f_{\mathrm{ES}}^{e, h\left(E_{i}\right)}\left[1-f_{\mathrm{GS}}^{e, h\left(E_{i}\right)}\right]}{\tau_{0}}-\frac{f_{\mathrm{GS}}^{e\left(E_{i}\right)} f_{\mathrm{GS}}^{h\left(E_{i}\right)}}{\tau_{r}} \\
& -\frac{f_{\mathrm{GS}}^{e, h\left(E_{i}\right)}\left[1-f_{\mathrm{ES}}^{e, h\left(E_{i}\right)}\right]}{\tau_{\mathrm{GS}}^{e, h\left(E_{i}\right)}}, \\
\frac{d f_{\mathrm{ES}}^{e, h\left(E_{i}\right)}}{d t}= & \frac{f_{\mathrm{res}}^{e, h}\left[1-f_{\mathrm{ES}}^{e, h\left(E_{i}\right)}\right]}{4 \tau_{c}}-\frac{f_{\mathrm{ES}}^{e, h\left(E_{i}\right)}\left[1-f_{\mathrm{GS}}^{e, h\left(E_{i}\right)}\right]}{\tau_{0}} \\
& -\frac{f_{\mathrm{ES}}^{e\left(E_{i}\right)} f_{\mathrm{ES}}^{h\left(E_{i}\right)}}{\tau_{r}}+\frac{f_{\mathrm{GS}}^{e, h\left(E_{i}\right)}\left[1-f_{\mathrm{ES}}^{e, h\left(E_{i}\right)}\right]}{2 \tau_{\mathrm{GS}}^{e, h\left(E_{i}\right)}-\frac{f_{\mathrm{ES}}^{e, h\left(E_{i}\right)}}{\tau_{\mathrm{ES}}^{e, h\left(E_{i}\right)}}}
\end{aligned}
$$

Here, the radiative lifetime is set to be $\tau_{r}=1 \mathrm{~ns}$, identical for carriers on the GS and the ES. Relaxation from ES to GS is accounted for by the time constant $\tau_{0}$ and the capture mechanism from the WL continuum into the QDs by the time constant $\tau_{c}$. For simplicity, the values of $\tau_{0}$ and $\tau_{c}$ are set to be identical for electrons and holes (these assumptions are discussed in the following). Thermal escape from GS and from ES is described by the time constants $\tau_{\mathrm{GS}}^{e, h\left(E_{i}\right)}$ and $\tau_{\mathrm{ES}}^{e, h\left(E_{i}\right)}$, respectively. The WL continuum acts as a carrier reservoir, which is approximated here by a single discrete energy state corresponding to the spontaneous emission peak of the WL continuum at $E_{\mathrm{WL}}=1.2 \mathrm{eV}$. All sets of rate equations for the QD distribution functions are coupled via two equations for the number of electrons/holes in the reservoir $f_{\text {res }}^{e, h}$ :

$$
\begin{aligned}
\frac{d f_{\mathrm{res}}^{e, h}}{d t}= & G-\frac{1}{N_{D}} \sum_{E_{i}} \frac{f_{\mathrm{res}}^{e, h}}{\tau_{c}} \cdot N_{D}^{E_{i}}\left[1-f_{\mathrm{ES}}^{e, h\left(E_{i}\right)}\right] \\
& +\frac{1}{N_{D}} \sum_{E_{i}} \frac{4 N_{D}^{E_{i}} f_{\mathrm{ES}}^{e, h\left(E_{i}\right)}}{\tau_{\mathrm{ES}}^{e, h\left(E_{i}\right)}}-\frac{f_{\mathrm{res}}^{e} f_{\mathrm{res}}^{h}}{\tau_{r}},
\end{aligned}
$$

where $G$ denotes the number of injected carriers per QD and per carrier lifetime $\tau_{r}$. Note here that $f_{\text {res }}^{e, h}$ are not the electron/ hole population probabilities, but the total number of carriers in the reservoir divided by the total number of QDs $N_{D}$.

Various experimental studies ${ }^{18,19}$ reveal that carrier dynamics in InAs QDs is dominated by Auger mechanisms and therefore dependent on the size of electron/hole densities. However, in Ref. 6 it has been shown that the experimental behavior of GS and ES threshold currents can be quantitatively reproduced by a constant (phenomenological) relaxation time as a single fitting parameter. Thus we believe a constant relaxation time is a reasonable approximation in the limited carrier density range considered here. In the subse-

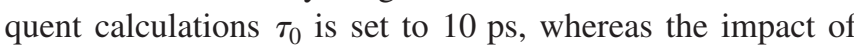
the capture time $\tau_{c}$ is discussed in the following. The time constants for the escape of electrons and holes can be derived from Eqs. (1) and (3) by assuming that the system reaches thermal equilibrium in the absence of external excitation:

$$
\begin{aligned}
& \tau_{\mathrm{GS}}^{e, h\left(E_{i}\right)}=\frac{\tau_{0}}{2} \exp \left[\frac{s^{e, h}\left(E_{i}^{\mathrm{ES}}-E_{i}\right)}{k_{B} T}\right], \\
& \tau_{\mathrm{ES}}^{e, h\left(E_{i}\right)}=4 \tau_{c} \exp \left[\frac{s^{e, h}\left(E_{\mathrm{WL}}-E_{i}^{\mathrm{ES}}\right)}{k_{B} T}\right] .
\end{aligned}
$$

In agreement with recent calculations ${ }^{20}$ we attribute a factor of $s^{e}=0.8$ of the measured transition energy-level

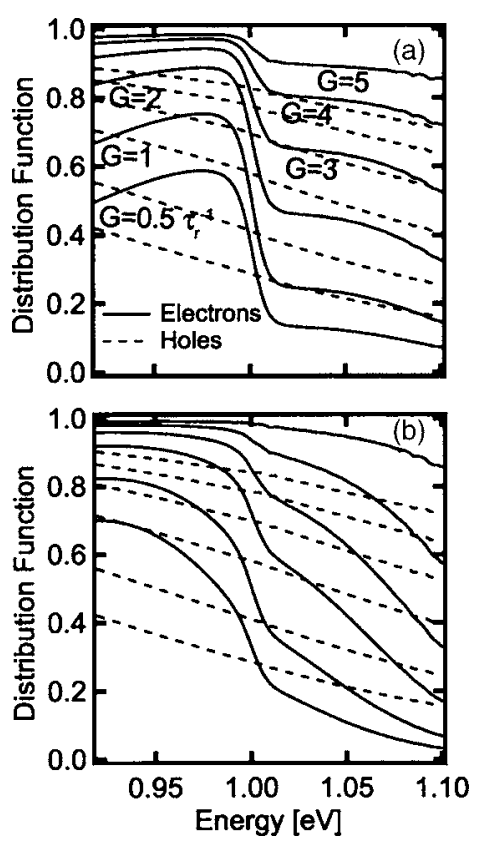

FIG. 4. Calculated carrier distribution functions for a DOS shown in Fig. 3(a) for electrons (full line) and holes (dashed lines) and at two different values for the capture time, $\tau_{c}=10$ ps [Fig. 4(a)] and $\tau_{c}=1$ ps [Fig. 4(b)].

separations $\left(E_{i}^{\mathrm{ES}}-E_{i}\right.$ and $\left.E_{\mathrm{WL}}-E_{i}^{\mathrm{ES}}\right)$ to the electron-level separations, and the remainder $s^{h}=0.2$ to the hole-level separations. Note here that the GS and ES escape times do not only depend on temperature $T$ and on the respective GS/ES transition energies $E_{i}$ and $E_{i}^{\mathrm{ES}}$, but also on relaxation time $\tau_{0}$ and capture time $\tau_{c}$, respectively. Since the escape times are crucial parameters for the results in this letter, a detailed derivation is given in the Appendix. Figures 3(b) and 3(c) depict the variation of escape times over energy for electrons (full line) and holes (dashed line). The energy dependence of GS escape times [Fig. 3(c)] stems from the variation in the ES-GS energy difference and favors the GS escape in lowerenergy QDs. Inversely, escape from ES to the discrete reservoir level [Fig. 3(b)] is favored for higher-energy QDs.

At first we discuss the impact of the capture time $\tau_{c}$ on the calculated results. The calculated carrier distribution functions for two different values $\tau_{c}=10 \mathrm{ps}$ and $\tau_{c}=1 \mathrm{ps}$ are plotted in Figs. 4(a) and 4(b), respectively. The full lines refer to the electron distributions and the dashed lines to the hole distributions. For a longer capture time $\left[\tau_{c}=10 \mathrm{ps}\right.$, Fig. $4(\mathrm{a})]$ and at low injection rates $\left(0<G<2 \tau_{r}^{-1}\right)$ most electrons reside in the $\mathrm{GS}$ levels ( $E<1 \mathrm{eV}$, full lines). In this regime QDs with different GS transition energies are not filled equally, due to the larger energy separation of GS and ES for QDs with a higher transition energy, resulting in a smaller escape time [see Fig. 3(c)] and in an enhanced occupation of the ES. When the GS starts to saturate $\left(G \geqslant 2 \tau_{r}^{-1}\right)$, Pauli blocking becomes important and the ES becomes increasingly populated $(E>1 \mathrm{eV})$. The overall behavior is determined by a slow-escape mechanism from the ES to the WL, which does not allow different QDs to "communicate" with each other, and a strong nonequilibrium carrier distribution is established. In contrast, the hole distribution function [Fig. 4(a), dashed lines] resembles very closely a Fermi distribution at all injection currents, and we conclude that the holes 

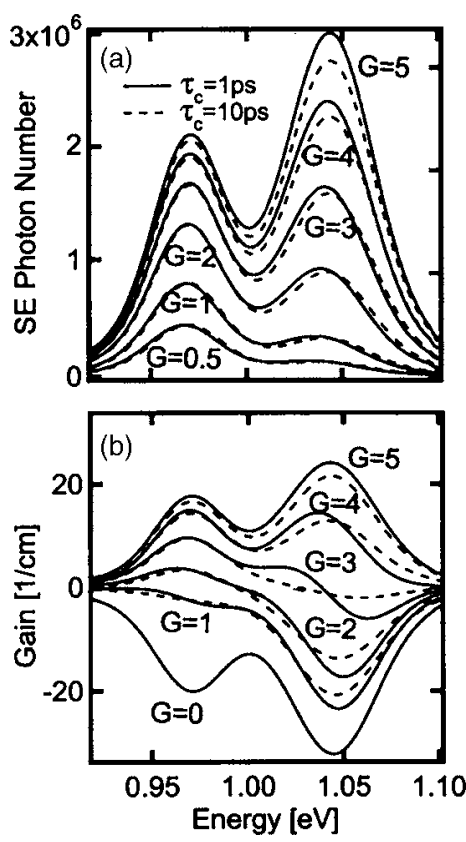

FIG. 5. Calculated level populations (a) and gain distributions (b) assuming $\tau_{c}=1 \mathrm{ps}$ (full lines) and $\tau_{c}=10 \mathrm{ps}$ (dashed lines). Note that the gain is shifted towards the low-energy side with respect to the population function. This shift depends on temperature, capture time, and carrier injection.

are in a situation close to quasithermal equilibrium due to a valence-band spacing that is inferior to $k_{B} T$. As a consequence, the assumption of a faster capture mechanism $\left(\tau_{c}\right.$ $=1 \mathrm{ps}$ ) does not change the overall behavior of the hole distribution function [Fig. 4(b), dashed lines]. In contrast the enhanced carrier-transfer process among QDs in the conduction band (full lines) leads to equilibration within the inhomogeneous broadening of the QD ensemble and also overall between GS and ES. As a result a probability distribution more similar to a Fermi function is established.

The impact of the different electron distributions for different capture times on the spontaneous emission and gain spectrum is displayed in Figs. 5(a) and 5(b), respectively. The full lines refer to calculations assuming $\tau_{c}=1 \mathrm{ps}$ and the dashed lines assuming $\tau_{c}=10 \mathrm{ps}$. In the calculation of SE and gain homogeneous energy broadening has to be considered. This is done by a convolution with a Lorentzian function with a width of $8 \mathrm{meV}$ (the technical details are discussed in the following paragraph). Note in Fig. 5(a) that the maximum position of GS and ES SE peaks shifted towards lower energies for a faster capture time $\tau_{c}=1 \mathrm{ps}$ (full lines) as compared to the peak values of the DOS [Fig. 3(a)]. This shift is due to carrier thermalization when the occupation of lowerenergy QDs is favored. Note also that in the corresponding gain curves in Fig. 5(b) the respective shifts are larger. At an injection rate of $G=4 \tau_{r}^{-1}$, ES gain reaches the peak value of the nearly saturated GS gain. This situation is particularly important for the interpretation of the experimental spectra, since both GS and ES lasing are expected to set in at around this gain value. Here the peak positions of GS population and GS gain nearly coincide with the DOS peak position, since the ground states of all QDs are completely filled. In contrast, excited states of the QD ensemble are far from being saturated, and thermal redistribution towards lower energies produces a redshift of the population and gain spectra. This effect is more pronounced for the gain curve, since population inversion is facilitated for QD families with a lower areal density.

It is important to note that the spectral redshift of gain and population functions only depends on the amount to which thermal equilibrium is established, and therefore indirectly on the chosen value of the capture time $\tau_{c}$. Assuming a slower capture time $\tau_{c}=10 \mathrm{ps}$ gain and population peaks are nearly centered at the peak positions of the DOS at all injection currents [Figs. 5(a) and 5(b), dashed lines]. In order to reproduce the spectral redshift of the gain curve at $T=300 \mathrm{~K}$, the assumption of a capture time $\tau_{c} \approx 1 \mathrm{ps}$ is essential.

\section{LASING SPECTRA}

In order to include carrier-photon interaction into the model, homogeneous energy broadening has to be considered, which arises due to dephasing processes. One of them is intrinsic dephasing, imposing a limit in the $\mu \mathrm{eV}$ range for typical radiative lifetimes around $1 \mathrm{~ns}$. Indeed ultralong dephasing times in the time range of several hundred picoseconds have been found at low temperatures. ${ }^{21,22}$ At room temperature the homogeneous line broadening increases to typically about $10-20 \mathrm{meV} \cdot{ }^{23,24}$ In this case other dephasing mechanisms have to be considered, such as phonon scattering or carrier-carrier scattering. ${ }^{23}$ The spectral lineshape of the homogenous broadening at an energy $E_{i}$ is described by a Lorentzian function over all discretized energies $E_{k}$ :

$$
L_{E_{k}}^{E_{i}}=\frac{\Gamma}{\pi} \cdot \frac{1}{\left(E_{i}-E_{k}\right)^{2}+\Gamma^{2}},
$$

where $\Gamma$ is the exciton homogeneous linewidth. An absorption/stimulated emission term can then be introduced in the sets of rate equations (1) and (2) by performing a convolution of inhomogeneous and homogeneous lineshapes:

$$
-B\left[f_{\mathrm{GS}, \mathrm{ES}}^{e\left(E_{i}\right)}+f_{\mathrm{GS}, \mathrm{ES}}^{h\left(E_{i}\right)}-1\right] \sum_{E_{k}} L_{E_{k}}^{E_{i}} N_{D}^{E_{k}} P_{\mathrm{GS}, \mathrm{ES}}^{E_{k}},
$$

with the respective photon numbers $P_{\mathrm{GS}(\mathrm{ES})}^{E_{k}}$ at the GS/ES transition energies. They are described by an additional set of two rate equations at each photon energy:

$$
\begin{aligned}
\frac{d P_{\mathrm{GS}}^{E_{i}}}{d t}= & -\frac{P_{\mathrm{GS}}^{E_{i}}}{\tau_{\varphi}}+\frac{2 \beta}{\tau_{r} N_{D}^{E_{i}} \sum_{E_{k}}} L_{E_{k}}^{E_{i}} N_{D}^{E_{k}} f_{\mathrm{GS}}^{e\left(E_{k}\right)} f_{\mathrm{GS}}^{h\left(E_{k}\right)} \\
& +2 B P_{\mathrm{GS}}^{E_{i}} \sum_{E_{k}} L_{E_{k}}^{E_{i}} N_{D}^{E_{k}}\left[f_{\mathrm{GS}}^{e\left(E_{k}\right)}+f_{\mathrm{GS}}^{h\left(E_{k}\right)}-1\right], \\
\frac{d P_{\mathrm{ES}}^{E_{i}}}{d t}= & -\frac{P_{\mathrm{ES}}^{E_{i}}}{\tau_{\varphi}}+\frac{4 \beta}{\tau_{r} N_{D}^{E_{i}} \sum_{E_{k}}} L_{E_{k}}^{E_{i}} N_{D}^{E_{k}} f_{\mathrm{ES}}^{e\left(E_{k}\right)} f_{\mathrm{ES}}^{h\left(E_{k}\right)} \\
& +4 B P_{\mathrm{ES}}^{E_{i}} \sum_{E_{k}} L_{E_{k}}^{E_{i}} N_{D}^{E_{k}}\left[f_{\mathrm{ES}}^{e\left(E_{k}\right)}+f_{\mathrm{ES}}^{h\left(E_{k}\right)}-1\right] .
\end{aligned}
$$

The photon lifetime $\tau_{\varphi}$ and the radiative transition probability $B$ have been derived from the experimental laser characteristics. ${ }^{6}$ More precisely, $\tau_{\varphi}$ is determined by the mir- 


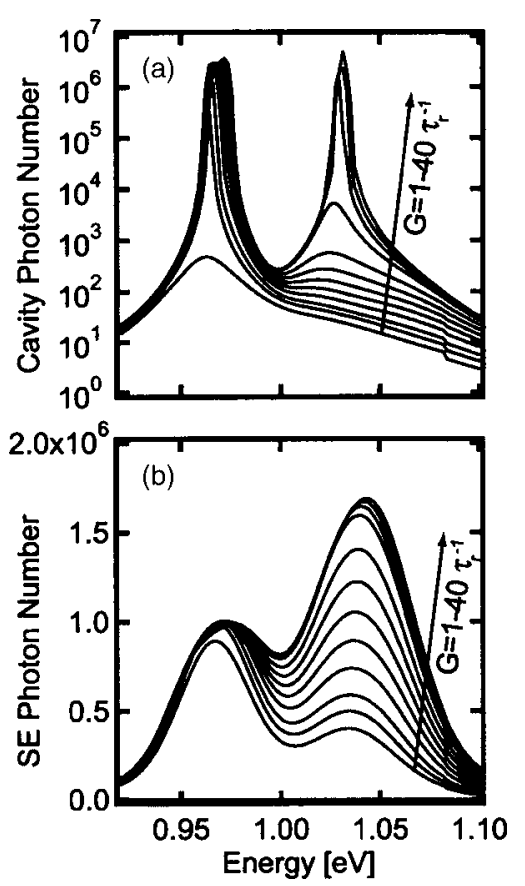

FIG. 6. Calculated spectra of the cavity photon number (a) and the spontaneously emitted photon number (b) in a $l=2 \mathrm{~mm}$ laser cavity at increasing carrier injection rate $G$. The FWHM of the homogenous broadening is fixed to $\Gamma=8 \mathrm{meV}$ and the assumed capture time is $\tau_{c}=1 \mathrm{ps}$.

ror and internal loss, whereas the $B$ factor accounts for the experimentally determined saturation of GS gain. The spontaneous emission coupling factor $\beta$, quantifying the fraction of spontaneously emitted photons that couple into the lasing mode, is set to a typical value of $10^{-4}$. The values of $\tau_{c}$ $=1 \mathrm{ps}$ and $\Gamma=8 \mathrm{meV}$ are used as fitting parameters to reproduce the experimental spectra.

We first analyze the spectral positions of the SE spectrum and lasing line. A detailed calculation of the spectrally resolved behavior is shown in Figs. 6(a) and 6(b). Figure 6(a) depicts the calculated cavity photon numbers corresponding to a spectrum from the facets in the experiment [see Fig. 1(a)]. Figure 6(b) shows the calculated spontaneous emission photon numbers according to the experiment of detected EL from holes [see Fig. 1(b)]. A comparison of Figs. 6(a) and 6(b) shows that GS and ES lasing emissions are redshifted from the respective energy positions of the spontaneous emission peak, where the ES lasing line shows a large shift $(\sim 15 \mathrm{meV})$ and the GS lasing line shows a small shift $(\sim 3 \mathrm{meV})$, in good agreement with the experimental spectra in Figs. 1(a) and 1(b). This behavior is understood in terms of the spectral shift of the gain curve with respect to the population curve, as discussed in the previous section (see Fig. 5). The size of this shift depends on the extent of state filling. GS population is nearly saturated at the GS threshold and the peak position of gain and population spectrum is similar. In contrast ES population is far off from being saturated at the ES threshold and the gain peak is shifted to the low-energy side of the population spectrum.

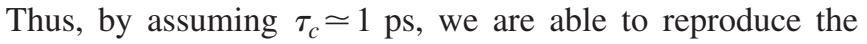
experimental shift of $\sim 20 \mathrm{meV}$ of the ES lasing line with respect to the population peak.

Now we discuss the impact of the homogeneous line-
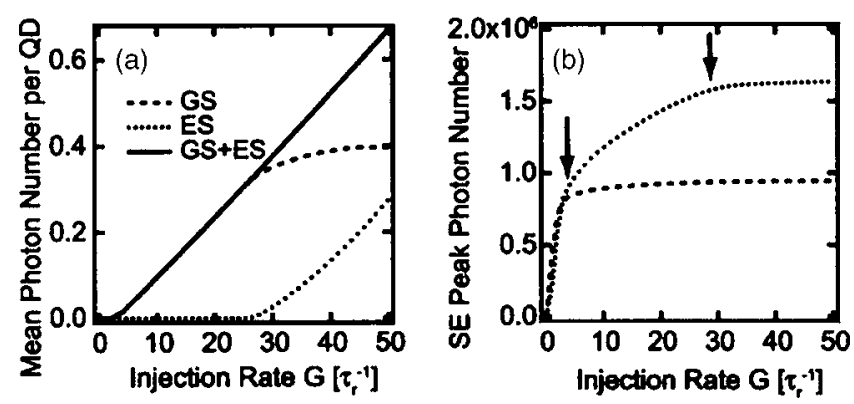

FIG. 7. Corresponding curves to Figs. 6(a) and 6(b) for the integrated cavity photon numbers (normalized by the total number of QDs) (a) and the values of the SE spectrum at the DOS peak position (b) plotted over the carrier injection rate $G$.

width on the (incomplete) population clamping at the threshold. The values of the GS/ES spontaneous emissions at the DOS peak positions over $G$ are displayed in Fig. 7(b). In these calculations, as in the experiment [see Fig. 2(b)], the level population of GS and ES increases above the respective threshold, although the GS population increases only very slightly. This increase can be adjusted using $\Gamma$ as a fitting parameter. For smaller values (e.g., $\Gamma=5 \mathrm{meV}$ ) the increase is much more pronounced, whereas a larger value (e.g., $\Gamma$ $=15 \mathrm{meV}$ ) leads to complete population clamping. That is because the extent to which QDs with energies away from the gain peak contribute to the laser modes in the gain center is governed by the homogenous linewidth $\Gamma$. A smaller value for $\Gamma$ leads to a smaller contribution to stimulated emission of QDs with energies off the lasing line. Consequently, the population in those QDs is not clamped at the threshold.

This has two more consequences: (1) The lasing line broadens with increasing carrier injection, as observed in the experiment [Fig. 1(a)] and also by other groups. ${ }^{25}$ We note that the larger broadening of the GS lasing line suggests a smaller value of $\Gamma$ for the GS-our simplified assumption of a constant value for $\Gamma$ indeed neglects the detailed dependence on the electronic structure and the injected carrier density. $^{23,24}$ (2) The GS lasing photon number does not completely saturate [Fig. 7(a)], as observed in the experiment [Fig. 2(a)] and in contrast to the prediction of a single-mode model. ${ }^{6}$ The reason is that the ES threshold, i.e., the current at which the ES starts to emit at the expense of the GS intensity, is reached gradually with the broadening of the ES lasing line.

Note that not only the homogeneous linewidth, but also the degree of carrier thermalization have a crucial impact on the behavior of population clamping above the threshold. Indeed, enhanced thermal coupling among different QDs leads to a larger number of carriers contributing to the stimulated emission process. It has been checked that for the same value of $\Gamma=8 \mathrm{meV}$, the assumption of very fast escape and capture times $\left(\tau_{c} \sim 100 \mathrm{fs}\right)$ lead to complete population clamping at the ES lasing threshold and results in complete saturation of both the GS lasing emission and GS/ES spontaneous emissions. Thus the opposite observation in the experiment [Figs. 2(a) and 2(b)] indicates that thermal equilibrium within the QD ensemble is not fully established. An assumption of a capture time $\tau_{c}$ in the range of $\approx 1 \mathrm{ps}$ is 


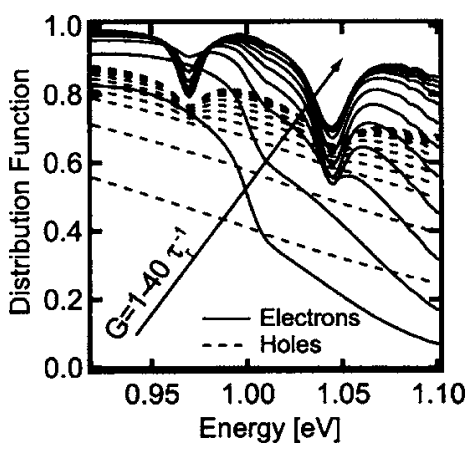

FIG. 8. Calculated carrier distribution functions under lasing conditions for electrons (full line) and holes (dashed lines).

therefore essential to reproduce on one hand the spectral shift of the lasing line and on the other hand the incomplete saturation of GS lasing intensities.

After having validated our model parameters by fitting the experiment, we can now calculated the carrier distribution functions under lasing conditions (Fig. 8), as compared to the nonlasing regime considered in Fig. 4. Under stimulated emission conditions the electron distributions (full lines) deviate even more severely from a quasi-Fermi distribution due to the effect of spectral hole burning. This is produced by a carrier-transfer process among different QDs that is not able to follow the much faster stimulated-emission mechanism. With increasing carrier injection the "spectral hole" increases without decreasing the net gain value, since an increasing number of QDs off the gain peak (and within the homogeneous broadening) contributes to the stimulated emission process. This effect is less pronounced in the hole distribution (dashed lines) due to a faster carrier transfer in the closely spaced valence-band states. We stress that the assumption of faster relaxation and capture rates in the valence band would even further confirm the establishment of a distribution close to thermal equilibrium in the valence band. In this case, the SE and gain spectra would be mainly determined by the electron distribution, and an estimation of capture and relaxation times would apply to electrons only.

\section{CONCLUSION}

In summary, the spectral investigation of the lasing mode and the spontaneous emission allows us to shed light on the interaction of different QDs. In fact, both thermal escape and homogeneous broadening allow the interaction among otherwise isolated QDs with important consequences on the laser characteristics.

Two main experimental findings are reported: a spectral redshift of the ES lasing line as compared to the population peak and incomplete clamping of GS and ES populations at threshold. The spectral redshift can be accounted for in a rate-equation model by a redshift of the ES gain peak, which is explained by a partial carrier redistribution to lowerenergy states in the inhomogeneously broadened QD ensemble. The incomplete clamping of GS and ES populations at threshold is attributed to a homogeneous linewidth that is inferior to the inhomogeneous broadening and limits the quantum efficiency of such a device. As a consequence the lasing line broadens above the respective threshold current and the GS emission power does not completely saturate at the ES threshold. The values of $\Gamma=8 \mathrm{meV}$ for the homogeneous broadening and $\tau_{c} \simeq 1$ ps for the capture time result in a good agreement with the experiment. The application of these values in the model and the ensemble of experimental observations indicate that a situation close to thermal equilibrium is established in the valence band, while strongly nonthermal electron distributions appear in the conduction band due to a larger energy spacing.

As a main conclusion, modeling in a rate-equation model including inhomogeneous and homogeneous energy broadenings not only provides a qualitative understanding of the experimentally found spontaneous emission spectra, but also allows us to estimate two key material parameters, i.e., the homogeneous linewidth $\Gamma$ and the capture time $\tau_{c}$. Both play an important role in the static and dynamic characteristics of QD lasers and amplifiers.

\section{ACKNOWLEDGMENTS}

This work was funded by the Swiss National Science Foundation, the Commission for Technology and Innovation (TOPNANO21 program), and the SER-COST program.

\section{APPENDIX: DERIVATION OF ESCAPE TERMS}

In Sec. III the escape terms from GS and ES $\left[\tau_{\mathrm{GS}}^{e, h\left(E_{i}\right)}\right.$ and $\left.\tau_{\mathrm{ES}}^{e, h\left(E_{i}\right)}\right]$ were given by assuming a thermal equilibrium in the absence of external excitation and interband recombination. In this case rate equation (1) can be written as

$$
\begin{aligned}
& \frac{\partial f_{\mathrm{GS}}^{e, h\left(E_{i}\right)}}{\partial t}=-\frac{f_{\mathrm{GS}}^{e, h\left(E_{i}\right)}\left(1-f_{\mathrm{ES}}\right)}{\tau_{\mathrm{GS}}^{e, h\left(E_{i}\right)}}+\frac{2 f_{\mathrm{ES}}^{e, h\left(E_{i}\right)}\left[1-f_{\mathrm{GS}}^{e, h\left(E_{i}\right)}\right]}{\tau_{0}}=0 \\
& \Rightarrow-\frac{f_{\mathrm{GS}}^{e, h\left(E_{i}\right)}}{\tau_{e}^{\mathrm{GS}}}+\frac{f_{\mathrm{GS}}^{e, h\left(E_{i}\right)} f_{\mathrm{ES}}^{e, h\left(E_{i}\right)}}{\tau_{\mathrm{GS}}^{e, h\left(E_{i}\right)}}+\frac{2 f_{\mathrm{ES}}^{e, h\left(E_{i}\right)}}{\tau_{0}}-\frac{2 f_{\mathrm{GS}}^{e, h\left(E_{i}\right)} f_{\mathrm{ES}}^{e, h\left(E_{i}\right)}}{\tau_{0}}=0
\end{aligned}
$$

Under the condition that the Fermi level $E_{f}$ of the system is inside the band gap $\left(f_{\mathrm{ES}} \ll 1, f_{\mathrm{GS}} \ll 1\right)$, Eq. (3) yields

$$
-\frac{f_{\mathrm{GS}}^{e, h\left(E_{i}\right)}}{\tau_{\mathrm{GS}}^{e, h\left(E_{i}\right)}}+\frac{2 f_{\mathrm{ES}}^{e, h\left(E_{i}\right)}}{\tau_{0}}=0 \Rightarrow \frac{f_{\mathrm{ES}}^{e, h\left(E_{i}\right)}}{f_{\mathrm{GS}}^{e, h\left(E_{i}\right)}}=\frac{\tau_{0}}{2 \tau_{\mathrm{GS}}^{e, h\left(E_{i}\right)}} \text {. }
$$

In this case $f_{\mathrm{GS}}$ and $f_{\mathrm{ES}}$ can be approximated by a Boltzmann distribution:

$$
\begin{aligned}
& f_{\mathrm{GS}}^{e, h\left(E_{i}\right)}=\exp \left(-\frac{s^{e, h} E_{i}-E_{f}}{k_{B} T}\right), \\
& f_{\mathrm{ES}}^{e, h\left(E_{i}\right)}=\exp \left(-\frac{s^{e, h} E_{i}^{\mathrm{ES}}-E_{f}}{k_{B} T}\right), \\
& \Rightarrow \frac{f_{\mathrm{ES}}^{e, h\left(E_{i}\right)}}{f_{\mathrm{GS}}^{e, h\left(E_{i}\right)}}=\exp \left[-\frac{s^{e, h}\left(E_{i}^{\mathrm{ES}}-E_{i}\right)}{k_{B} T}\right]=\frac{\tau_{0}}{2 \tau_{\mathrm{GS}}^{e, h\left(E_{i}\right)}},
\end{aligned}
$$

which finally yields the GS escape time:

$$
\tau_{\mathrm{GS}}^{e, h\left(E_{i}\right)}=\frac{\tau_{0}}{2} \exp \left[\frac{s^{e, h}\left(E_{i}^{\mathrm{ES}}-E_{i}\right)}{k_{B} T}\right] .
$$


The same considerations $\left(f_{\text {res }} \ll 1, f_{\mathrm{ES}} \ll 1\right)$ are taken into account in the sum of rate equations (1) and (2), in such a way that a detailed balance between capture and remission is established:

$$
\begin{aligned}
& \frac{f_{\text {res }}\left(1-f_{\mathrm{ES}}^{E_{i}}\right)}{4 \tau_{c}}-\frac{f_{\mathrm{ES}}^{E_{i}}}{\tau_{\mathrm{ES}}^{e, h\left(E_{i}\right)}}=0 \\
& \Rightarrow \frac{f_{\text {res }}}{f_{\mathrm{ES}}}=\exp \left[-\frac{s^{e, h}\left(E_{\mathrm{WL}}-E_{i}^{\mathrm{ES}}\right)}{k_{B} T}\right]=\frac{4 \tau_{c}}{\tau_{\mathrm{ES}}^{e, h}\left(E_{i}\right)},
\end{aligned}
$$

which yields the ES escape time:

$$
\tau_{\mathrm{ES}}^{e, h\left(E_{i}\right)}=4 \tau_{c} \exp \left[\frac{s^{e, h}\left(E_{\mathrm{WL}}-E_{i}^{\mathrm{ES}}\right)}{k_{B} T}\right] .
$$

${ }^{1}$ Y. Arakawa and H. Sakaki, Appl. Phys. Lett. 40, 939 (1982).

${ }^{2}$ X. Huang, A. Stintz, C. P. Hains, G. T. Liu, J. Cheng, and K. J. Malloy, IEEE Photonics Technol. Lett. 12, 227 (2000).

${ }^{3}$ O. B. Shchekin and D. G. Deppe, Appl. Phys. Lett. 80, 3277 (2002).

${ }^{4}$ P. Bhattacharya and S. Ghosh, Appl. Phys. Lett. 80, 3482 (2002).

${ }^{5}$ H. Saito, K. Nishi, A. Kamei, and S. Sugou, IEEE Photonics Technol. Lett. 12, 1298 (2000).

${ }^{6}$ A. Markus, J. X. Chen, O. Gauthier-Lafaye, J. G. Provost, C. Paranthoën, and A. Fiore, IEEE J. Sel. Top. Quantum Electron. 82, 1308 (2003).

${ }^{7}$ H. Jiang and J. Singh, J. Appl. Phys. 85, 7438 (1999).
${ }^{8}$ H. Huang and D. G. Deppe, IEEE J. Quantum Electron. 37, 691 (2001). ${ }^{9}$ M. Grundmann, O. Stier, S. Bognaár, C. Ribbat, F. Heinrichsdorff, and D. Bimberg, Phys. Status Solidi A 178, 255 (2000).

${ }^{10}$ H. D. Summers, J. D. Thomson, P. M. Smowton, P. Blood, and M. Hopkinson, Semicond. Sci. Technol. 16, 140 (2001).

${ }^{11}$ A. Sakamoto and M. Sugawara, IEEE Photonics Technol. Lett. 12, 107 (2000).

${ }^{12}$ M. Grundmann, Appl. Phys. Lett. 77, 1428 (2000).

${ }^{13}$ M. Sugawara, N. Hatori, H. Ebe, M. Ishida, Y. Arakawa, T. Akiyama, K. Otsubo, and Y. Nakata, J. Appl. Phys. 97, 043523 (2005).

${ }^{14}$ P. Blood, A. I. Kucharska, J. P. Jacobs, and K. Griffiths, J. Appl. Phys. 70, 1144 (1991).

${ }^{15}$ A. Markus and A. Fiore, Phys. Status Solidi A 201, 338 (2004).

${ }^{16}$ O. B. Shchekin and D. G. Deppe, Appl. Phys. Lett. 80, 2758 (2002).

${ }^{17}$ A. A. Dikshit and J. M. Pikal, Appl. Phys. Lett. 82, 4812 (2003).

${ }^{18}$ T. Müller, W. Parz, F. F. Schrey, G. Strasser, and K. Unterrainer, Semicond. Sci. Technol. 19, S278 (2004).

${ }^{19}$ D. Morris, N. Perret, and S. Fafard, Appl. Phys. Lett. 75, 3593 (1999).

${ }^{20}$ A. J. Williamson, L. W. Wang, and Alex Zunger, Phys. Rev. B 62, 12963 (2000).

${ }^{21}$ P. Borri, W. Langbein, S. Schneider, U. Woggon, R. L. Sellin, D. Ouyang, and D. Bimberg, Phys. Rev. Lett. 87, 157401 (2001).

${ }^{22}$ D. Birkedal, K. Leosson, and J. M. Hvam, Phys. Rev. Lett. 87, 227401 (2001).

${ }^{23}$ P. Borri, W. Langbein, J. Mørk, J. M. Hvam, F. Heinrichsdorff, M.-H. Mao, and D. Bimberg, Phys. Rev. B 60, 7784 (1999).

${ }^{24}$ M. Bayer and A. Forchel, Phys. Rev. B 65, 041308(R) (2002).

${ }^{25}$ H. Shoji, Y. Nakata, K. Mukai, Y. Sugiyama, M. Sugawara, N. Yokoyama, and H. Ishikawa, Appl. Phys. Lett. 71, 193 (1997). 